\title{
Queixas osteomusculares dos trabalhadores e condições biomecânicas no trabalho em metalúrgica de alumínio
}

\author{
Worker's musculoskeletal complaints and \\ biomechanical work conditions in an aluminium smelter
} João Silvestre Silva-Junior', Gustavo Pontes Buzzoni², Luiz Carlos Morrone ${ }^{1}$

\begin{abstract}
RESUMO | Introdução: O alumínio é um metal proveniente da bauxita, sendo o Brasil um importante produtor mundial. Estudos descrevem distúrbios osteomusculares relacionados ao trabalho entre trabalhadores que transformam o alumínio em produto para consumo. Objetivo: Avaliar as condições ergonômicas e as queixas osteomusculares em trabalhadores metalúrgicos de alumínio. Métodos: Estudo transversal realizado em indústria transformadora de alumínio na cidade de Embu das Artes, São Paulo. Foram aplicados checklists para a avaliação simplificada do risco de lombalgia e para a avaliação simplificada do risco de distúrbios musculoesqueléticos de membros superiores relacionados ao trabalho no setor de coquilha da empresa. Os 38 trabalhadores do setor responderam ao Questionário Nórdico de Sintomas Osteomusculares (QNSO). Resultados: O posto de trabalho apresenta um risco moderado para agravamento/desencadeamento de lombalgia e distúrbios musculoesqueléticos de membros superiores relacionados ao trabalho. Nos últimos 12 meses, 15,8\% dos trabalhadores se queixaram de dores lombares, e, nos últimos sete dias, 5,3\% relataram queixas de dores em punhos/mãos/dedos. Conclusão: Condições biomecânicas do trabalho podem estar relacionadas ao desenvolvimento de queixas osteomusculares. A gestão dos riscos ocupacionais nesse ramo industrial se faz necessária para promover produtividade e qualidade de vida entre os trabalhadores.
\end{abstract}

Palavras-chave I metalurgia; riscos ocupacionais; saúde do trabalhador; medicina do trabalho; ergonomia; sistema osteomuscular.

ABSTRACT | Background: Aluminum is a metal commonly found in bauxite ore and Brazil is a major producer worldwide. Studies describe work-related musculoskeletal disorders among workers in the aluminum industry. Objectives: Evaluating ergonomic conditions and musculoskeletal complaints among workers in an aluminum smelter. Methods: Cross-sectional study carried out in an aluminum smelter in Embu das Artes, São Paulo. Checklists were applied to simplify evaluation of the risk of lower back pain and the risk of musculoskeletal disorders of the upper limbs related to work in a specific sector. The 38-sector workers answered to the Nordic Musculoskeletal Questionnaire (NMQ). Results: The ergonomic conditions present moderate risk for lower back pain and upper limb disorders. In the last 12 months, $15.8 \%$ of workers complained of back pain and in the last seven days, $5.3 \%$ of workers reported complaints of pain in wrists/hands/fingers. Conclusion: Biomechanical conditions of work can be related to musculoskeletal complaints. The management of occupational hazards in this branch of industry is needed to promote productivity and quality of life among workers.

Keywords I metallurgy; occupational risks; occupational health; occupational medicine; human engineering; musculoskeletal system.

Trabalho realizado na Faculdade de Ciências Médicas da Santa Casa de São Paulo (FCMSCSP) - São Paulo (SP), Brasil.

'Departamento de Saúde Coletiva, FCMSCSP - São Paulo (SP), Brasil.

${ }^{2}$ Centro Tecnológico da Marinha - São Paulo (SP), Brasil.

in memoriam.

DOI: 10.5327/Z1679-443520160215 


\section{INTRODUÇÃO}

A indústria do alumínio tem recebido importantes investimentos, tanto da esfera pública quanto da privada ao redor do mundo. Este metal é extraído da bauxita, e o Brasil é um importante produtor mundial deste metal, que é extraído da bauxita ${ }^{1,2}$. Os lingotes de alumínio e seus elementos metálicos podem ser processados em laminadoras, forjas e fundições. Os produtos finais são utilizados na indústria naval, elétrica, automobilística, aeroespacial e de construção civil; indústria leve para utensílios domésticos, equipamentos de oficina e joias; fabricação de fios e cabos condutores de estruturas para janelas, telhados e revestimentos ${ }^{2}$.

Trabalhadores da indústria de transformação do alumínio relataram queixas osteomusculares em estudos realizados nos Estados Unidos da América ${ }^{3}$, na Noruega ${ }^{4}$ e no Irã ${ }^{5}$. Comparando os trabalhadores que fazem atividades manuais de produção com os administrativos/técnicos/gerentes, os primeiros apresentaram mais queixas ${ }^{4} \mathrm{e}$ um maior risco para curto e longo afastamento do trabalho em função de distúrbios musculoesqueléticos ${ }^{6}$. Fatores relacionados à biomecânica do trabalho foram descritos, sendo associados aos quadros osteomusculares $^{3,7}$.

Na Noruega, os distúrbios osteomusculares representaram $40 \%$ dos dias de trabalho perdidos entre metalúrgicos analisados no setor de alumínio 4 . Para os casos de longo afastamento do trabalho por doença, a legislação previdenciária brasileira reconhece que há nexo técnico epidemiológico previdenciário entre a Classificação Nacional de Atividade Econômica (CNAE) das empresas metalúrgicas de alumínio e o intervalo M40-M54 do capítulo XIII da Classificação Estatística Internacional de Doenças e Problemas Relacionados à Saúde (CID-10). Em outras palavras, os benefícios previdenciários decorrentes de quadros de dorsopatias entre os trabalhadores dessa categoria industrial são caracterizados como relacionados ao trabalho ${ }^{8}$.

Considerando a falta de evidências na literatura científica brasileira sobre queixas ortopédicas em trabalhadores do ramo industrial de transformação do alumínio, este estudo pretendeu identificar as condições biomecânicas para a execução do trabalho em setor operacional de metalúrgica de alumínio e queixas ortopédicas relatadas pelos trabalhadores expostos.

\section{MÉTODOS}

Estudo observacional, transversal e descritivo realizado em uma empresa metalúrgica transformadora de alumínio, localizada no município de Embu das Artes, São Paulo. Fundada em 1974, utiliza alumínio primário para a fabricação de conexões e eletrodutos, com uma equipe composta de 474 funcionários. Em avaliação qualitativa prévia, o setor de coquilha foi considerado como aquele que apresentava as piores condições biomecânicas. Os 38 trabalhadores deste setor foram convidados a participar da pesquisa e todos aceitaram o convite. A coleta de dados foi realizada no ano de 2012.

As condições biomecânicas do posto de trabalho foram analisadas conforme duas ferramentas do tipo checklist, que vêm sendo utilizadas em estudos brasileiros sobre a saúde dos trabalhadores ${ }^{9-11}$ :

1. avaliação simplificada do risco de lombalgia ${ }^{12}$ — composta de 13 perguntas com resposta dicotômica (sim/não), cuja soma foi interpretada da seguinte forma: 0 a 3 pontos (altíssimo risco de lombalgia); 4 a 5 pontos (alto risco de lombalgia); 6 a 7 pontos (risco moderado de lombalgia); 8 a 10 pontos (baixo risco de lombalgia); 11 a 13 pontos (baixíssimo risco de lombalgia);

2. avaliação simplificada do risco de ocorrência de distúrbios musculoesqueléticos de membros superiores relacionados ao trabalho ${ }^{12}$ - composta de 26 perguntas com resposta dicotômica (sim/não), cuja soma foi interpretada da seguinte forma: 0 a 3 pontos (ausência de fatores biomecânicos); 4 a 6 pontos (fator biomecânico pouco significativo); 7 a 9 pontos (fator biomecânico de moderada importância); 10 a 14 pontos (fator biomecânico significativo); 15 pontos ou mais (fator biomecânico muito significativo).

Em ambos os checklists, a situação negativa gera pontuação para interpretação da condição biomecânica.

No momento em que vinham para a consulta ocupacional periódica, os participantes respondiam a uma versão em português, do Brasil, do Questionário Nórdico de Sintomas Osteomusculares (QNSO) (NordicMusculoskeletal Questionnaire-NMQ) ${ }^{13}$.Essa ferramenta foi desenvolvida para padronizar a mensuração de queixas do sistema osteomuscular e facilitar a comparação de resultados. São procedidas perguntas acerca de ocorrência de 
dores nas diversas regiões anatômicas do corpo (pescoço, ombro, cotovelo, antebraço, punhos/mãos/dedos, região dorsal, quadril/coxa, joelho, tornozelo/pé) nos últimos 7 dias e nos últimos 12 meses, com resposta binária ( $\operatorname{sim} /$ não).

Os resultados do QNSO demonstram a incidência percentual de queixas no grupo estudado e foram apresentadas, por seguimento analisado, tantonos últimos sete dias quanto no último ano.

O estudo foi aprovado pelo Comitê de Ética em Pesquisa em Seres Humanos da Irmandade da Santa Casa de Misericórdia de São Paulo (Parecer no 38/2012).

\section{RESULTADOS}

\section{AVALIAÇÃO DO LOCAL DE TRABALHO}

O fluxo de produção inicia-se com o encaminhamento da matéria-prima - o alumínio sólido - para fornos, onde o material é derretido a uma temperatura aproximada de $650^{\circ} \mathrm{C}$. O alumínio líquido pode seguir dois caminhos: coquilha ou injetora. No primeiro o processo, o caminho é manual, com despejo do alumínio derretido em um molde, chamado coquilha, que posteriormente será rebarbado para a retirada da peça. No processo automatizado pela injetora, a peça é desmoldada em uma prensa. Após a produção da peça, realizada por um dos dois processos, ela é encaminhada para a usinagem (apara das arestas), tamboreadeira (polimento, brilho e limpeza), montagem (colocação de insumos como parafusos e vedação nas peças), embalagem e expedição.

A coquilha é um grande molde metálico que possui duas peças que formam a parte principal do molde, além de outras quatro pequenas partes. Com uso de uma concha, o trabalhador derrama o alumínio derretido no forno para dentro do molde. Após solidificação, a peça em estado bruto é retirada da coquilha para ser encaminhada ao setor de rebarba. O ciclo de trabalho, de aproximadamente um minuto, é finalizado com a remontagem da coquilha após retirada da peça.

Com relação ao checklist para a avaliação simplificada do risco de lombalgia, houve perda de pontuação nos quesitos: posicionamento estático do tronco em posição fletida entre $30^{\circ}$ e $60^{\circ}$; esforço com ferramenta ou com as mãos estando o tronco encurvado; necessidade de manusear cargas que estejam longe do tronco e também em posição assimétrica; necessidade de ficar constantemente com os braços longe do tronco em posição suspensa; trabalho requer que o trabalhador fique na posição estática sem apoio; trabalho exige rotação do tronco. A soma do escore alcançou seis pontos, o que, de acordo com o critério de interpretação, é um risco moderado de lombalgia.

O checklist para a avaliação simplificada do risco de distúrbios musculoesqueléticos de membros superiores relacionados ao trabalho pontuou negativamente os seguintes quesitos: trabalho não pode ser feito sem que haja contato da mão ou punho, ou dos tecidos moles com alguma quina viva de objeto ou ferramenta; a tarefa não pode ser feita sem o uso de luvas; aparentemente as mãos não fazem pouca força; a força de compressão exercida pela mão não é pequena; ocorre esforço estático do braço e do pescoço na realização do trabalho; trabalho não é feito sem a extensão ou flexão do punho; ocorre abdução do braço acima de $45^{\circ}$ ou elevação dos braços acima do nível dos ombros como rotina; posto não permite flexibilidade no posicionamento de ferramentas, dispositivos ou componentes; altura do posto não é regulável; não há rodízios nas tarefas; uma mesma tarefa é realizada por um mesmo trabalhador durante mais de quatro horas por dia. A pontuação do escore foi de oito pontos, cuja interpretação é de um fator biomecânico de moderada importância.

Ao se visitar o ambiente de trabalho, nota-se uma desorganização no aproveitamento do espaço com depósitos de matérias em áreas de circulação. Desta forma, há dificuldade para a passagem dos trabalhadores, o que pode propiciar traumas e quedas. $\mathrm{O}$ manuseio recorrente do metal líquido aquecido durante o trabalho pode proporcionar queimaduras de pele. $\mathrm{O}$ uso de ferramentas e o manuseio da coquilha podem gerar trauma de mãos, ou até mesmo queda sobre os pés. E, ainda, isso pode ser agravado pela repetitividade e monotonia das tarefas.

São fornecidas, como medida de proteção individual, vestimentas individuais para a proteção contra queimaduras do corpo e calçados com biqueira de aço para proteger qualquer queda de objeto sobre os pés.

\section{AVALIAÇÃO DOS TRABALHADORES}

Foram avaliados 38 funcionários, sendo uma população composta exclusivamente por homens. A idade variou de 21 a 56 anos, com mediana de 34 anos e uma média de 34,5 anos. O tempo de trabalho variou de 3 meses a 17 anos, com uma média pouco superior a três anos.

Conforme a Tabela 1, nota-se que a grande maioria dos trabalhadores não apresenta queixas osteomusculares crônicas ou agudas. Entre aqueles que apresentaram queixas nos últimos 12 meses, houve maior relato de dorsalgias. Em relação aos quadros agudos, a incidência mais elevada foi a de dores em punhos, mãos e dedos. 
Tabela 1. Distribuição das queixas osteomusculares dos trabalhadores do setor de coquilha da indústria de alumínio nos últimos 7 dias e nos últimos 12 meses, conforme região anatômica do corpo. São Paulo, 2012 (n=38).

\begin{tabular}{lcc|c|c}
\multirow{2}{*}{ Região de dor } & \multicolumn{2}{c|}{ Últimos 7 dias } & \multicolumn{2}{c}{ Últimos 12 meses } \\
\cline { 2 - 5 } & $n$ & $\%$ & $n$ & $\%$ \\
\hline Pescoço & 0 & 0,0 & 2 & 5,3 \\
\hline Ombros & 1 & 2,6 & 2 & 5,3 \\
\hline Cotovelo & 0 & 0,0 & 0 & 0,0 \\
\hline Antebraço & 0 & 0,0 & 0 & 0,0 \\
\hline Punhos/mãos/dedos & 2 & 5,3 & 1 & 2,6 \\
\hline Região dorsal & 1 & 2,6 & 6 & 15,8 \\
\hline Quadris e/ou coxas & 0 & 0,0 & 0 & 0,0 \\
\hline Joelhos & 0 & 0,0 & 0 & 0,0 \\
\hline Tornozelo e/ou pés & 0 & 0,0 & 0 & 0,0 \\
\hline Sem queixas & 34 & 89,5 & 27 & 71,1 \\
\hline
\end{tabular}

\section{DISCUSSÃO}

A biomecânica do posto de trabalho estudado mostrou-se com risco moderado tanto para membros superiores quanto para lombalgia. A repercussão dessa situação foi encontrada nas queixas apresentadas pelos trabalhadores, visto a incidência de dores osteomusculares crônicas no último ano. A repercussão foi menos intensa nos últimos sete dias, o que significa uma sobrecarga insidiosa com repercussão a médio/longo prazo.

Assim como em nosso estudo, o relato de lombalgia foi a queixa crônica mais frequente entre trabalhadores deste ramo produtivo no Irã e nos Estados Unidos da América ${ }^{3,5}$. A condição biomecânica de carregar peso no trabalho é um fator de risco para dor lombar crônica ${ }^{7}$. A prevenção de quadros de dor lombar é muito importante, pois a lombalgia é preditora do absenteísmo de curto e médio prazo ${ }^{6}$.

Este estudo demonstrou menor frequência de sintomas osteomusculares em trabalhadores da indústria de alumínio, comparando com o estudo norte-americano ${ }^{4}$. Essa realidade pode ser influenciada em função do perfil do grupo brasileiro ser mais jovem e com menos tempo de trabalho na empresa do que o grupo americano. Sabe-se que tais condições são fatores de risco para distúrbios osteomusculares ${ }^{4,7}$. Cabe considerar que não houve confidencialidade no preenchimento do questionário. Isso pode representar um viés de aferição pela possibilidade de o trabalhador omitir suas queixas, com o intuito de minimizar o impacto negativo de sua imagem junto ao empregador.

Na Noruega, a maior prevalência de queixas osteomusculares entre trabalhadores manuais do setor produtivo e a associação desse desfecho com o tempo na função sugere que o ambiente de trabalho esteja associado ao problema ${ }^{4}$. Portanto, situações com potencial lesivo, como as encontradas neste estudo no Brasil, devem ter sua condição ergonômica estudada e reconhecida nos Programas de Controle Médico de Saúde Ocupacional (PCMSO) ${ }^{14}$. Além disso, sabe-se que o aumento da idade é um fator de risco para dor lombar crônica, devendo, assim, haver uma redução gradual da exposição a cargas ergonômicas? .

O delineamento transversal descritivo do estudo, que se propõe a levantar hipóteses, é limitado. Portanto, não cabe inferir associação ou causalidade entre as variáveis sociodemográficas ocupacionais e as queixas osteomusculares.

\section{CONCLUSÕES}

Este estudo demonstra que, no setor de coquilha de uma metalúrgica produtora de peças de alumínio, há postos de trabalho com risco moderado para o desenvolvimento de distúrbios musculoesqueléticos. Portanto, o nexo causal pode ser considerado para quadros ortopédicos que acometam trabalhadores expostos a tais condições biomecânicas.

A presença de queixas osteomusculares relatadas pelos trabalhadores devem ter sua etiologia investigada para o estabelecimento de medidas de proteção e prevenção, tanto de agravamento dos quadros quanto de desencadeamento em população susceptível.

A exposição a condições inadequadas de trabalho, que são descritas nesse tipo de processo produtivo em países de diferentes condições de desenvolvimento socioeconômico, devem ser estudadas. Sugerimos ações de adaptação do ambiente e condições de trabalho às capacidades e necessidades do trabalhador com regulagem do mobiliário, fornecimento de cadeiras/bancos reguláveis, instituição de rodízio entre postos de trabalho e pausas regulares. Medidas eficazes de proteção à saúde dos trabalhadores podem minimizar o absenteísmo ou presenteísmo e, assim, estimular a qualidade de vida no trabalho. 


\section{REFERÊNCIAS}

1. CiccantellP.Globalizaçãoedesenvolvimento baseadoemmatérias-primas: o caso da indústria do alumínio. Novos Cadernos NAEA. 2005;8(2):41-72.

2. Norberg G. Metales: Propiedades Quimicas y Toxicidad. In: Enciclopedia de Salud y Seguridad en el Trabajo. Madrid: OIT, 2008. p. 63.2-63.3.

3. Hughes RE, Silverstein BA, Evanoff BA. Risk factors for work-related musculoskeletal disorders in an aluminum smelter. Am J Ind Med. 1997;32(1):66-75.

4. Morken T, Moen B, Riise T, Bergum O, Bua L, Hauge SH, et al. Prevalence of musculoskeletal symptoms among aluminium workers. Occup Med. 2000;50(6):414-21.

5. Aghilinejad M, Mousavi SA, Nouri MK, Ahmadi AB. Work-related musculoskeletal complaints among workers of Iranian aluminum industries. Arch Environ Occup Health. 2012;67(2):98-102.

6. Morken T, Riise T, Moen B, Hauge SH, Holien S, Langedrag A, et al. Low back pain and widespread pain predict sickness absence among industrial workers. BMC Musculoskelet Disord. 2003;4:21.

7. Silva MC, Fassa AG, Valle NC. Dor lombar crônica em uma população adulta do Sul do Brasil: prevalência e fatores associados. Cad. Saúde Pública. 2004;20(2):377-85.

8. Brasil. Decreto no 3.048, de 06 de maio de 1999. Aprova o Regulamento da Previdência Social, e dá outras providências. Diário Oficial da União, Brasília, DF, 07 maio 1999.
9. Maciel DP, Millen RA, Xavier CA, Morrone LC, Silva-Júnior JS Musculoeskeletal disorder related to the work of doctors who perform medical invasive evaluation. Work. 2012;41:1860-3.

10. Sacouche DA, Morrone LC, Silva-Júnior JS. Impact of ergonomics risk among workers in clothes central distribution service in a hospital. Work. 2012;41:1836-40.

11. Silva-Júnior JS, Correa LR, Morrone LC. Evaluation of lumbar overload in hotel maids. Work. 2012;41:2496-8.

12. Couto HA. Ergonomia aplicada ao trabalho: manual técnico da máquina humana. Belo Horizonte: Ergo, 1995.

13. Pinheiro FA, Tróccoli BT, Carvalho CV. Validação do Questionário Nórdico de Sintomas Osteomusculares como medida de morbidade. Rev Saúde Pública. 2002;36(3):307-12.

14. Brasil. Ministério do Trabalho e Emprego. Normas regulamentadoras de segurança e saúde do trabalho. NR 7 - Programa de Controle Médico de Saúde Ocupacional. Brasília, DF: MTE, 1978.

Endereço para correspondência: João Silvestre Silva-Junior - Rua Dr. Cesário Mota Jr., 61, $6^{\circ}$ andar - CEP 01221-020 - São Paulo (SP), Brasil -

E-mail: silvajunior.js@gmail.com 